\title{
Early Warning System Design of Safe Production of Coal Mine Based on Improved BP Neural network
}

\author{
Qiu Hong-Li ${ }^{1, a}$, SUN Pei-shuo ${ }^{2, b}$ \\ 1,2 Henan Quality Engineering Vocational College, Pingdingshan HeNan 467001,China \\ a zcysym@126.com, ${ }^{b}$ djxzcy@126.com
}

Keywords: BP neural network, improved algorithm, safe production of coal mine, early warning

\begin{abstract}
For staff, environment, equipment and management the four aspects, the early warning index system of coal mine safety production adopts the additional momentum method and adaptive vector, particle swarm optimization (PSO) algorithm and the method of variable weight and asynchronous learning factor and other measures. It is to optimize the BP neural network model and apply the model to the early warning system of coal mine safety, and at the same time, it also compared with other neural network model. The simulation result shows that the recognition accuracy of improved BP neural network model is higher than PSO-BP model, the model not only can effectively reduce the possibility of a network get into a local minimum point, but also have the characteristics of fast convergence speed, high accuracy and etc., and it can provide a scientific basis to the coal mine production safety early warning management.
\end{abstract}

\section{Introduction}

Coal mine production is a kind of high-risk production operation with high frequency of production accidents. The early warning mechanism for production safety is a very important measure to strengthen the prior control of coal mine safety. For these years, there are many literatures about early warning research in the field of coal mine safety production, for example one literature proposes outburst early warning of coal and gas on the basis of geographic information system and the neural network. Early warning model mainly adopts the methods of clustering analysis and artificial neural network. But these research methods put more emphasis on warning index and the linear relationship between the objective function, slow convergence speed, which has some problems on local extremism points. For the existing problems, this paper puts forward a BP neural network model based on improved particle swarm, and this algorithm model can scientifically measure and determine the alert level of coal mine safety production, thus provide scientific basis for the early warning management of coal mine safety production.

\section{The PSO algorithm and improvement}

\section{The basic theory of particle swarm optimization (PSO)}

Particle swarm optimization (PSO) is based on swarm intelligence evolution algorithm. The basic idea is that PSO initializes a set of random factors to find the best solution through iteration in the group collaboration and information sharing between particles. During each iteration, the particles update themselves by tracking two "extreme", the first is the particles themselves to find the optimal solution and another extreme value is the optimal solution in the whole population.

Assuming that $\mathrm{D}$ dimension space sets up with $\mathrm{M}$ particles, which in the case of $\mathrm{i}$ position, $x i=\left(x_{i 1}, x_{i 2}, x_{i 3}, \ldots, x_{i d}\right)$, the particle speed $v i=\left(v_{i 1}, v_{i 2}, v_{i 3}, \ldots, v_{i d}\right)$, among which $\mathrm{i}=1,2,3 \ldots, \mathrm{M}$. The best position retrieved in history is pbest $=\left(p_{i 1}, p_{i 2}, p_{i 3}, \ldots, p_{i d}\right)$, the best post that retrieved in the whole article swarm is gbest $=\left(p_{g 1}, p_{g 2}, p_{g 3}, \ldots, p_{g d}\right)$.

$$
\begin{aligned}
& v(t+1)=\omega \times v(t)+c_{1} \times r_{1} \times(\operatorname{pbest}(t)-x(t))+c_{2} \times r_{2} \times(\text { gbest }(t)-x(t)) \\
& x(t+1)=x(t)+v(t+1)
\end{aligned}
$$

Among $x$ is Inertia weight; $c_{1}$ and $c_{2}$ is learning factors; $r_{1}$ and $r_{2}$ si random figures from 0 to 1 . 


\section{The methods of improved BP neural network}

There are many improved algorithms of BP neural network. In this paper, the improvement scheme combines early warning requirements of coal mine safety production:

(1) The traditional algorithm

Additional momentum method: not only has the effect of gradient, but also affects the change trend. It still needs to be considered in weight correction, it can ignore the small changes in the network. The adjustment formula is shown in figure 3 considering the additional power factors:

$$
\begin{aligned}
& \nabla w_{i j}(k+1)=(1-m c) \eta s_{i} p_{j}+m c \nabla w_{i j}(k) \\
& \Delta b_{i}(k+1)=(1-m c) \eta s_{i}+m c \Delta b_{i}(k)
\end{aligned}
$$

The conditions are as follows:

$$
m c=\left\{\begin{array}{lr}
0 & \mathrm{SSE}(\mathrm{k})>\mathrm{SSE}(\mathrm{k}-1) \times 1.04 \\
0.95 & \mathrm{SSE}(\mathrm{k})>\mathrm{SSE}(\mathrm{k}-1) \\
m c & \text { others }
\end{array}\right.
$$

Adaptive vector: the design of the adaptive vector function depends on the convergence of the training process of neural networks, so that it can reduce the oscillation phenomenon in the learning process, and improve the network speed. The calculation formula for improving learning vector is:

$$
h(k+1)=\left\{\begin{array}{cr}
1.05 h(k) & \mathrm{SSE}(\mathrm{k}+1)<\mathrm{SSE}(\mathrm{k}) \\
0.7 h(k) & \mathrm{SSE}(\mathrm{k}+1)>\mathrm{SSE}(\mathrm{k}) \times 1.04 \\
h(k) & \text { others }
\end{array}\right.
$$

(2) Improve the particle swarm algorithm BP

Even if the particle swarm optimization is easy to operate and has fast convergence rate, it still has the problems of low precision and local optimal solution easily capture. The method used for further improvement of the particle swarm optimization is to reduce the linear time-varying weight combined with the learning process of the asynchronous time change.

(1) Time-varying weight method

In general, the particle swarm has better ability to search the early stage of flight, especially flying ability with increased number of iterations. Hypothesizing the scope of inertia weight data is $\left[\omega_{\min }, \omega_{\max }\right]$, maximum iterations is inter-max, so the $t$ inertia weight: $\omega(t)=\omega_{\max }-\frac{\omega_{\max }-\omega_{\min }}{\text { nter }-\max } \times t$

(2) Asynchronous time-varying learning method

In order to strengthen the global search of the particle swarm in the early stages of the flight, to make the particle swarm converge to find the global optimal solution. Particle swarm in the early stage have weaker social learning ability, weak ability of autonomic learning, while the late can continuously reduce self learning factor $\mathrm{c} 1$ and increase social learning coefficient factor c1.

$$
\begin{aligned}
& c_{1}=\left(c_{1 f}-c_{1 i}\right) \times \frac{\text { inter }}{\text { inter }-\max }+c_{1 i} \\
& c_{2}=\left(c_{2 f}-c_{2 i}\right) \times \frac{\text { inter }}{\text { inter }-\max }+c_{2 i}
\end{aligned}
$$

\section{The set up of early warning system of improved BP neural network}

The application of BP neural network in early warning system of coal mine production safety gives full play of the advantages of nonlinear mapping and self-learning and adaptive ability of artificial neural network. To improve the intelligence through the optimization of BP neural network in order to avoid falling into local minimum and the defects of slow convergence speed. The basic idea is to put the early warning indicators of coal mine production safety into the neural network pretreatment, then improved particle swarm optimization (MPSO - BP) by improved BP (GBP), 
particle BP network training and testing swarm optimization (PSO and BP), BP neural network recognition. And then design warning intensity classification of coal mine safety production by using the algorithm. The early warning model of coal mine safety production is shown in Fig 1.

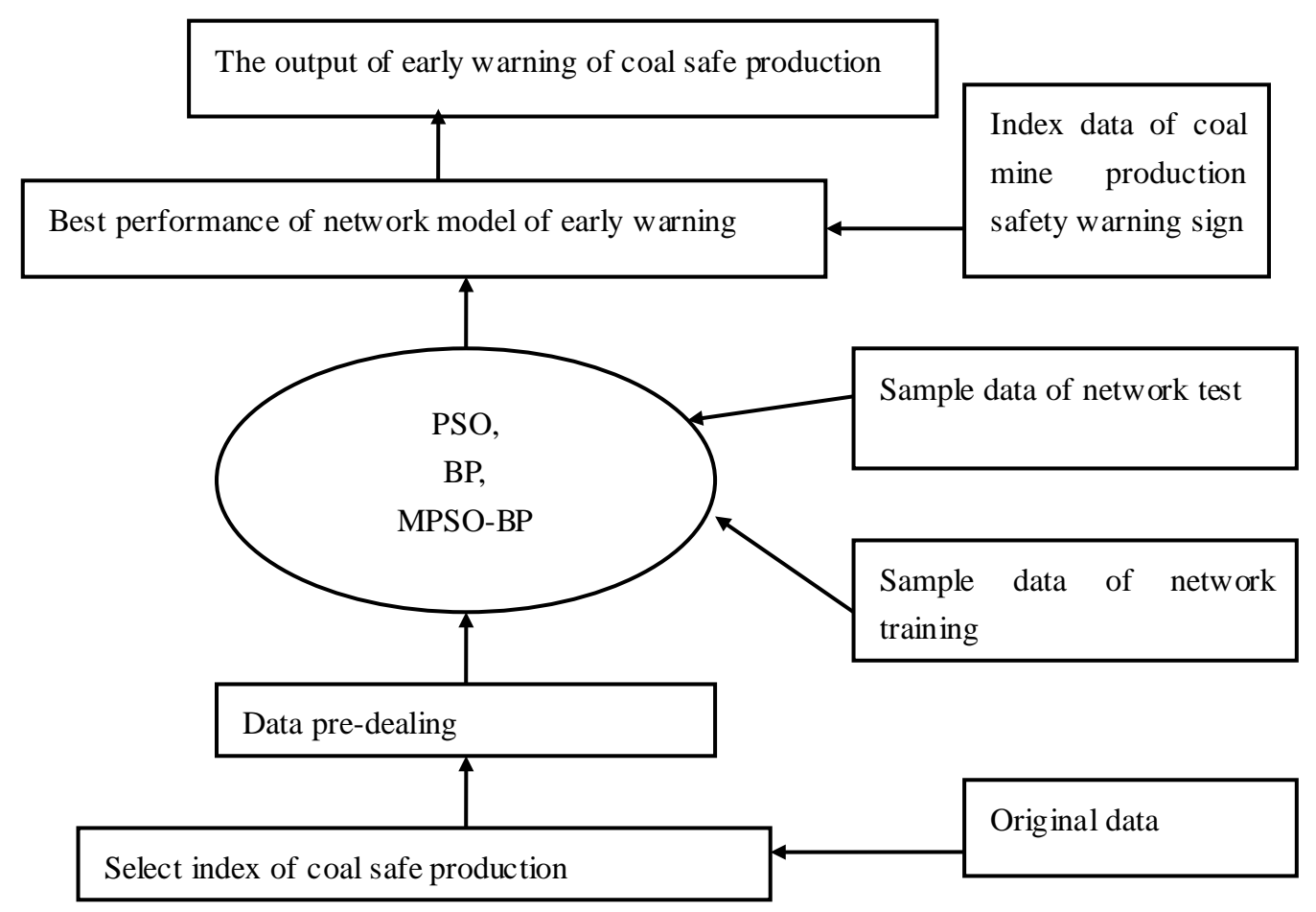

Fig 1: the model of early warning system of improved BP neural network

\section{The application of early warning system of improved BP neural network}

By comparing the two methods of the change in the fitness function value to reflect the recognition accuracy as can be shown in Fig 2. PSO-BP network fitness function stabilizes iterations, the function value is high. When the MPSO-BP neural network at 160 iteration times, the fitness function achieves stability, its function will be worth less than PSO-BP values. When set the neural network output error as fitness function, it has a larger output error. The identification precision of MPSO-BP neural network model is higher and more practical than PSO-BP model. So, we should choose the MPSO-BP model for coal mine safety early warning method, and calculate measurement index for safety warning alarm.

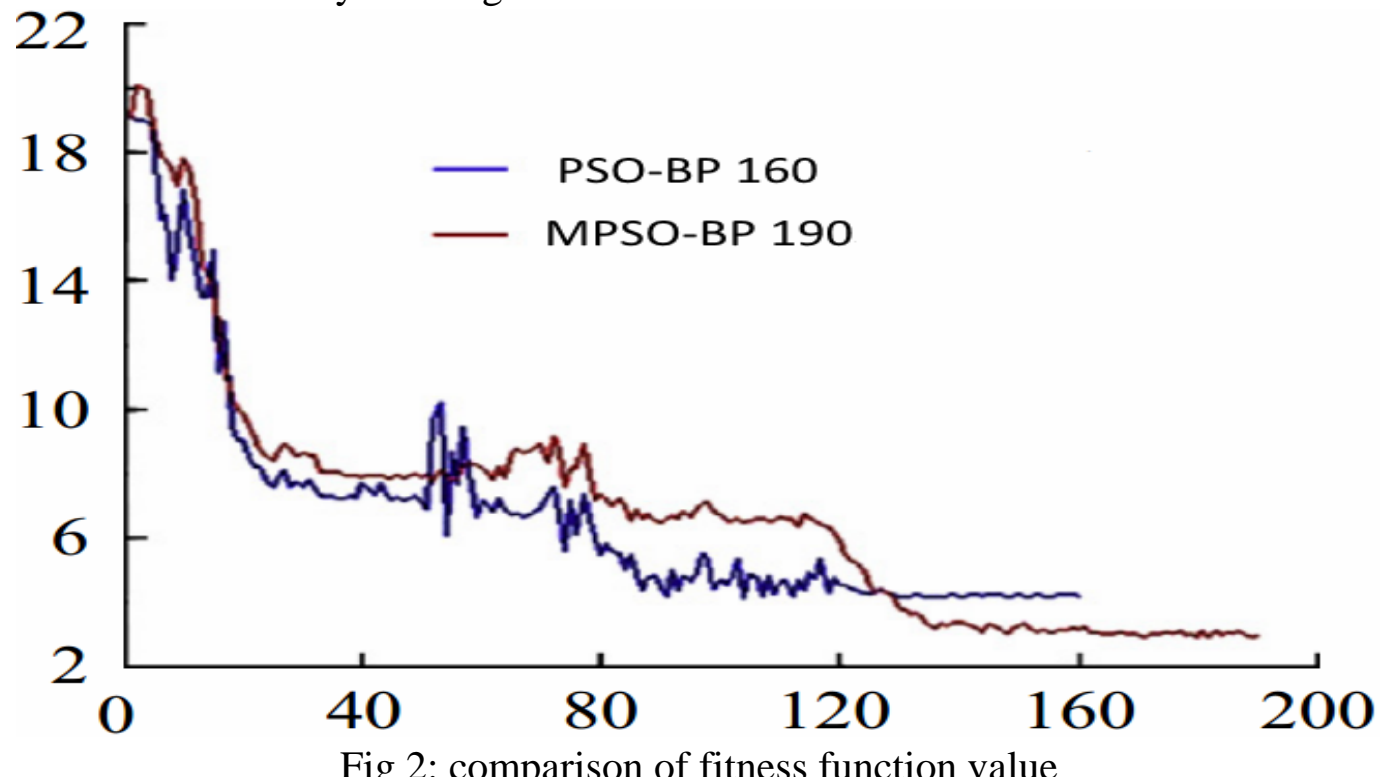


(1) Training and testing samples

Choose standardization of early warning indicators from 2008 to 2014, $\left[\mathrm{N}_{1}, \mathrm{~N}_{2}, \mathrm{~N}_{3}, \mathrm{~N}_{4}, \mathrm{~N}_{5}, \mathrm{~N}_{6}, \mathrm{~N}_{7}, \mathrm{~N}_{8}\right.$ ] as the training samples input. According to the enterprise security expert evaluation, we can judge the enterprise each year requires safety warning output $\left[\mathrm{T}_{1}\right.$, $\left.\mathrm{T}_{2}, \mathrm{~T}_{3}, \mathrm{~T}_{4}, \mathrm{~T}_{5}, \mathrm{~T}_{6}, \mathrm{~T}_{7}, \mathrm{~T}_{8}\right]$, and $\mathrm{T}_{1}=(01000), \mathrm{T}_{2}=(00100), \mathrm{T}_{3}=(00100), \mathrm{T}_{4}=(00100), \mathrm{T}_{5}=(00010)$, $\mathrm{T}_{6}=(00010), \mathrm{T}_{7}=(00010), \mathrm{T}_{8}=(00001)$, choose to enter $2012\left[\mathrm{~N}_{5}\right]$ and the desired output $\left[\mathrm{T}_{5}\right]=$ (00010) as the test sample.

(2) Neural network design

Input and output layer neurons of coal mine production safety early warning in the MPSO-BP model are 29 and 5 respectively. According to empirical formula and MATLAB program testing, the number of hidden layer neurons is 7, learning vector is 0.05 . Weighted factor of MPSO-BP $\varpi_{\text {max }}=0.9, \omega_{\min }=0.3$, learning factor $\omega_{\max }=0.9, c_{1 i}=2.5, c_{1 f}=0.5, \quad c_{2 i}=0.5, \quad c_{2 f}=2.5$. The particle swarm $M=40$, the maximum number of iteration is 1000 , particle velocity range is [0, 20]. Using the MATLAB program to run the program, the results are shown in table 1.

Table 1: analysis of error value of MPSO-BP neural network

\begin{tabular}{|l|l|l|l|}
\hline NO & simulation output & Expected output & Error value \\
\hline 1 & $(0.00000 .92000 .11000 .10000 .0481)$ & $(00100)$ & 0.0308 \\
\hline 2 & $(0.00010 .10920 .96060 .00620 .1207)$ & $(00100)$ & 0.0308 \\
\hline 3 & $(0.00000 .02980 .85240 .03100 .0786)$ & $(00100)$ & 0.0298 \\
\hline 4 & $(0.00000 .02980 .85240 .03100 .0786)$ & $(00100)$ & 0.0298 \\
\hline 5 & $0.85240 .03100 .0786)$ & $(00010)$ & 0.0298 \\
\hline 6 & $0.85240 .03100 .0786)$ & $(00010)$ & 0.0298 \\
\hline 7 & $0.85240 .03100 .0786)$ & $(00010)$ & 0.0298 \\
\hline 8 & $0.85240 .03100 .0786)$ & $(00001)$ & 0.0298 \\
\hline
\end{tabular}

\section{Summary}

This paper establishes the early warning index system of coal mine safety production from the four aspects: personnel, equipment, environment and management, and compares the application of GBP, PSO-BP, MPSO-BP neural network optimization model in coal mine production safety early warning condition. By comparing the three model simulation results we can know that the identification precision of MPSO-BP network model is higher than GBP model and PSO-BP model and its relative performance is the best. MPSO-BP network model can effectively reduce the possibility of falling into local minimum neural networks, the convergence speed, high accuracy, and can provide scientific basis for the safe production of coal mining enterprises early warning.

\section{References}

[1] Ma JJ, You JX,Chen Z.Gray neural network model based on modified particle swarm optimization algorithm and its application.[J].Tongji Univ (Nat Sci),2012,40(05):740-3.

[2] Sun Xiuying, Wang Qian,, Gas prediction and simulation research of coal mine based on the improved BP algorithm[J]. Coal Technology, 2014, 33(3):19-21.

[3] Gong Pei, Simulation research of safety evaluation model of the improved neural network of coal mine[J]. Computer Simulation, 2012, 29(1):156-158.

[4] Dai Honglei, Han Litao, Chen Hongfa, Gas disaster prediction model based on quasi-Newton optimization algorithm of BP neural network[J]. Coal Technology, 2008, 27(11):71-74.

[5] Han Chao, Che Yongcai, Wang Jibo, coal demand prediction model of the improved BP neural network[J]. Journal of Liaoning Technical University, 2005,24(S1):290-292. 